\title{
Effect of Weight Loss after Bariatric Surgery on Thyroid-Stimulating Hormone Levels in Patients with Morbid Obesity and Normal Thyroid Function
}

\author{
João Sérgio Neves ${ }^{1,2}$ (1) Sofia Castro Oliveira ${ }^{1,3} \cdot$ Pedro Souteiro $^{1,3} \cdot$ Jorge Pedro $^{1} \cdot$ \\ Daniela Magalhães ${ }^{1,3} \cdot$ Vanessa Guerreiro $^{1} \cdot$ Rita Bettencourt-Silva $^{1,3}$. \\ Maria Manuel Costa ${ }^{1,3}$. Ana Cristina Santos ${ }^{4,5}$. Joana Queirós ${ }^{1,6}$. Ana Varela ${ }^{1,3,6}$. \\ Paula Freitas ${ }^{1,3,6,7}$ - Davide Carvalho ${ }^{1,3,7}$ - AMTCO Group ${ }^{6}$
}

Published online: 19 July 2017

(C) Springer Science+Business Media, LLC 2017

\begin{abstract}
Background Several studies have reported that morbid obesity is associated with increased thyroid-stimulating hormone (TSH) levels. However, it is not clear what is the impact of bariatric surgery on postoperative thyroid function. The aim of this study was to evaluate the effect of weight loss after bariatric surgery on TSH levels in euthyroid patients with morbid obesity.
\end{abstract}

Methods We performed a retrospective observational study of 949 euthyroid patients $(86.1 \%$ female; age $42.0 \pm 10.3$ years, BMI $44.3 \pm 5.7 \mathrm{~kg} / \mathrm{m}^{2}$ ) with morbid obesity submitted to bariatric surgery (laparoscopic adjustable gastric band, Roux-en-Y gastric bypass, or sleeve gastrectomy). Patients were subdivided in two groups: normal TSH group (TSH $<2.5 \mathrm{mU} / \mathrm{L}$ ) and high-normal TSH group (TSH $\geq 2.5 \mathrm{mU} / \mathrm{L})$. The impact of anthropometric parameters, comorbidities,
João Sérgio Neves

joaosergioneves@gmail.com

Sofia Castro Oliveira

sofiacastro.oliveira@gmail.com

Pedro Souteiro

pedrobsouteiro@gmail.com

Jorge Pedro

jorgebraganca@gmail.com

Daniela Magalhães

danielascmagalhaes@gmail.com

Vanessa Guerreiro

vanessa.a.guerreiro@gmail.com

Rita Bettencourt-Silva

ritabettsilva@gmail.com

Maria Manuel Costa

maria_manuel_costa@hotmail.com

Ana Cristina Santos

acsantos@med.up.pt

Joana Queirós

joanaqr@gmail.com

\author{
Ana Varela \\ anavarelasande@gmail.com \\ Paula Freitas \\ paula_freitas@sapo.pt \\ Davide Carvalho \\ Davideccarvalho@gmail.com
}

1 Department of Endocrinology, Diabetes and Metabolism, Centro Hospitalar São João, Porto, Portugal

2 Department of Surgery and Physiology, Cardiovascular Research Center, Faculty of Medicine, University of Porto, Porto, Portugal

3 Faculty of Medicine of University of Porto, Porto, Portugal

4 Department of Clinical Epidemiology, Predictive Medicine and Public Health, University of Porto Medical School, Porto, Portugal

5 ISPUP-EPIUnit, Universidade do Porto, Porto, Portugal

6 Multidisciplinary Group for Surgical Management of Obesity, Centro Hospitalar São João, Porto, Portugal

7 Instituto de Investigação e Inovação em Saúde, University of Porto, Porto, Portugal 
TSH, free thyroxine (FT4), free triiodothyronine (FT3), type of surgery, and excessive body weight loss (EBWL) on TSH variation 12 months after surgery was evaluated.

Results The high-normal TSH group (24.3\% of patients) included more women, presented a higher BMI, higher systolic blood pressure, and higher FT3 levels. There was a significant decrease of TSH 12 months after surgery that was more marked in the high-normal TSH group (normal TSH group: $1.57 \pm 0.49$ to $1.53 \pm 0.69 \mathrm{mIU} / \mathrm{L}, p=0.063$; high-normal TSH group: $3.23 \pm 0.59$ to $2.38 \pm 0.86 \mathrm{mIU} / \mathrm{L}, p<0.001)$. In a multivariate analysis, after adjusting for relevant covariates, EBWL, baseline BMI, and baseline FT3 were significantly associated with TSH decrease 12 months after bariatric surgery.

Conclusion Bariatric surgery promotes a decrease of TSH that is significantly greater in patients with high-normal TSH and is independently associated with EBWL after surgery.

Keywords Morbid obesity - Thyroid function . Thyroid-stimulating hormone $\cdot$ Bariatric surgery

\section{Introduction}

Obesity is one of the most common diseases in developed countries, affecting more than 600 million adults worldwide [1]. It is associated with several comorbidities and endocrine abnormalities, including thyroid dysfunction [2]. The most effective treatment of obesity to date is bariatric surgery [3, 4]. Several comorbidities and endocrine abnormalities are known to reverse after bariatric surgery [3-6].

Thyroid hormone axis plays an important role in body weight balance, with hypothyroidism being associated with weight gain and hyperthyroidism promoting weight loss [2, 7]. Even within the normal range, variations of thyroid function have been associated with significant weight variation [8, 9]. On the other hand, obesity, particularly morbid obesity, has been associated with significant alterations of thyroid function $[10,11]$. Most studies reported increased serum thyroidstimulating hormone (TSH) concentrations in patients with morbid obesity $[10,11]$. The mechanism and the clinical implications of this alteration remains uncertain. Furthermore, the effect of bariatric surgery on postoperative thyroid function remains incompletely understood with previous studies showing contradictory results regarding the variation of TSH after bariatric surgery and the relation of TSH variation with postoperative weight loss [12-19].

Therefore, our aim was to evaluate the effect of weight loss after bariatric surgery on TSH variation in patients with morbid obesity and normal thyroid function.

\section{Materials and Methods}

\section{Study Design and Participants}

We performed a retrospective observational study evaluating patients with morbid obesity and normal preoperative thyroid function submitted to bariatric surgery in our institution between January 2010 and June 2015. Patients were excluded if they had a history of thyroid disease, treatment with thyroid hormone, antithyroid drugs, amiodarone or lithium, or if TSH or free thyroxine (FT4) was not within the normal reference range before the surgery (TSH $<0.35$ or $>4.94 \mathrm{mU} / \mathrm{L}$, or FT4 $<0.70$ or $>1.48 \mathrm{ng} / \mathrm{dL}$ ). Patients missing preoperative TSH or FT4 and those missing TSH 1 year after surgery were also excluded. Of the 1450 patients submitted to bariatric surgery in our institution during the study period, after applying the exclusion criteria, 949 patients were included in our analysis.

Patients were subdivided in two groups: normal TSH group that included patients with serum TSH levels below $2.5 \mathrm{mU} / \mathrm{L}$ and high-normal TSH group including those with a serum TSH level equal or superior to $2.5 \mathrm{mU} / \mathrm{L}$. This cutoff was selected based on previous reports that indicate that more than $95 \%$ of healthy individuals have TSH levels below $2.5 \mathrm{mU} / \mathrm{L}$ $[20,21]$.

\section{Clinical Parameters Evaluated}

The following preoperative parameters were evaluated: age, sex, body mass index (BMI), waist to hip ratio (WHR), blood pressure (BP), TSH, FT4, free triiodothyronine (FT3), history of diabetes, dyslipidemia and hypertension, and the type of bariatric surgery performed [laparoscopic adjustable gastric band (LAGB), Roux-en-Y gastric bypass (RYGB) or sleeve gastrectomy (SG)]. TSH serum levels 12 months after surgery and the excessive body weight loss in percentage (EBWL) 12 months after surgery were also evaluated.

Diabetes was defined by fasting plasma glucose $\geq 126 \mathrm{mg} /$ $\mathrm{dL}$, glycated hemoglobin $\geq 6.5 \%, 2$-h plasma glucose after a 75 -g oral glucose tolerance test $\geq 200 \mathrm{mg} / \mathrm{dL}$, or the use of antihyperglycemic drugs. Hypertension was defined as systolic $\mathrm{BP} \geq 140 \mathrm{mmHg}$, diastolic $\mathrm{BP} \geq 90 \mathrm{mmHg}$ or the use of antihypertensive drugs. Dyslipidemia was defined by the use of lipid-lowering agents, serum low-density lipoprotein (LDL) cholesterol $\geq 160 \mathrm{mg} / \mathrm{dL}$, serum high-density lipoprotein (HDL) cholesterol $<40 \mathrm{mg} / \mathrm{dL}$, or serum triglycerides $\geq 200 \mathrm{mg} / \mathrm{dL}$.

EBWL was calculated using the formula: [(preoperative weight-current weight) / (preoperative weight-ideal weight to produce BMI $\left.\left.25 \mathrm{~kg} / \mathrm{m}^{2}\right)\right] \times 100$.

TSH, FT4, and FT3 were measured on serum, obtained from blood samples during clinical evaluations, by 
chemiluminescence immunoassay on the Abbott Diagnostics Architect system (Abbott Diagnostics).

\section{Statistical Analysis}

For continuous variables, independent $t$ tests (for comparison between groups) or paired $t$ tests (for comparisons within the same group at different times) were performed. Differences between groups regarding categorical variables were evaluated with chi-squared test. To evaluate the effect of preoperative parameters on TSH variation after surgery, we used simple linear regression and multiple linear regression models. Results are presented as mean \pm standard deviation for continuous variables and as percentages for categorical variables. Statistical analyses were performed with Stata software, version 14.1 (StataCorp). We considered a two-sided $P$ value less than 0.05 to be statistically significant.

\section{Results}

\section{Baseline Population Characteristics}

Among the 949 patients analyzed, 86.1\% were female and the mean age was $42.0 \pm 10.3$ years. The patients presented a mean preoperative weight of $116.4 \pm 18.6 \mathrm{~kg}$, a mean preoperative BMI of $44.3 \pm 5.7 \mathrm{~kg} / \mathrm{m}^{2}$, and a waist circumference of $123.7 \pm 13.3 \mathrm{~cm}$ (Table 1 ). Thirty percent of the patients had diabetes, $44.1 \%$ dyslipidemia, and $60.6 \%$ hypertension. The baseline serum TSH level was $1.97 \pm 0.88 \mathrm{mIU} / \mathrm{L}$, FT4 was $1.05 \pm 0.13 \mathrm{ng} / \mathrm{dL}$, and FT3 was $3.20 \pm 0.45 \mathrm{pg} / \mathrm{mL}$. The surgical procedure most performed was RYGB (58.6\% of patients), followed by LAGB (20.9\%) and SG (20.6\%).

Seven hundred twenty-three patients $(75.7 \%)$ had a TSH level below $2.5 \mathrm{mU} / \mathrm{L}$ and were included in the normal TSH group. Two hundred thirty-two $(24.3 \%)$ were included in the high-normal TSH groups. The groups were not significantly different regarding most clinical and laboratorial parameters (Table 1), with exception of sex (92.6\% female in high-normal TSH group vs $84.0 \%$ in normal TSH group, $p=0.001$ ), BMI $\left(45.1 \pm 6.0 \mathrm{~kg} / \mathrm{m}^{2}\right.$ in high-normal TSH group vs $44.1 \pm 5.5 \mathrm{~kg} /$ $\mathrm{m}^{2}$ in normal TSH group, $p=0.016$ ), systolic blood pressure $(135.9 \pm 17.8 \mathrm{mmHg}$ in high-normal TSH group vs $132.4 \pm 17.3 \mathrm{~kg} / \mathrm{m} 2$ in normal TSH group, $p=0.011$ ), and FT3 $(3.35 \pm 0.47 \mathrm{pg} / \mathrm{mL}$ in high-normal TSH group vs $3.15 \pm 0.44 \mathrm{pg} / \mathrm{mL}$ in normal TSH group, $p<0.001)$.

\section{Weight Loss 12 months After Bariatric Surgery}

The mean EBWL 12 months after bariatric surgery was $69.5 \pm 25.6 \%$ and the mean BMI decrease after surgery was $13.19 \pm 5.38 \mathrm{~kg} / \mathrm{m}^{2}$. The weight loss after bariatric surgery was not significantly different between groups (EBWL,
$69.6 \pm 26.4 \%$ in normal TSH group vs $69.0 \pm 23.0 \%$ in high-normal TSH group, $p=0.736$; BMI decrease, $13.00 \pm 5.42 \mathrm{~kg} / \mathrm{m}^{2}$ in normal TSH group vs $13.75 \pm 5.21 \mathrm{~kg} / \mathrm{m}^{2}$ in high-normal TSH group, $\left.p=0.073\right)$.

Regarding the weight loss per type of bariatric surgery, RYGB was associated with significantly higher EBWL and BMI decrease than SG and LAGB $(p<0.001)$, and SG was associated significantly higher EBWL and BMI decrease than LAGB $(p<0.001)$. The mean EBWL was $79.1 \pm 19.4 \%$ for RYGB, $70.8 \pm 22.8 \%$ for $\mathrm{SG}$, and $40.4 \pm 22.1 \%$ for LAGB; and mean BMI decrease was $15.13 \pm 4.26 \mathrm{~kg} / \mathrm{m}^{2}$ for RYGB, $13.46 \pm 5.05 \mathrm{~kg} / \mathrm{m}^{2}$ for $\mathrm{SG}$, and $7.30 \pm 4.23 \mathrm{~kg} / \mathrm{m}^{2}$ for LAGB.

\section{TSH Variation 12 months After Bariatric Surgery}

We observed a significant decrease of TSH levels 12 months after surgery in the group of all patients. The mean TSH decreased from $1.97 \pm 0.88$ to $1.73 \pm 0.82 \mathrm{mIU} / \mathrm{L}(p<0.001)$. When evaluating by baseline TSH subgroup, the TSH decrease was significantly greater in the high-normal TSH group $(0.85 \pm 0.84 \mathrm{mIU} / \mathrm{L}$ decrease in the high-normal TSH group vs $0.05 \pm 0.62 \mathrm{mIU} / \mathrm{L}$ in the normal TSH group), and only in the high-normal TSH group this decrease was statistically significant (normal TSH group $1.57 \pm 0.49-1.53 \pm 0.69 \mathrm{mIU} / \mathrm{L}$, $p=0.063$; high-normal TSH group $3.23 \pm 0.59-2.38 \pm 0.86$ $\mathrm{mIU} / \mathrm{L}, p<0.001$ ) (Fig. 1).

\section{Predictors of TSH Decrease After Bariatric Surgery}

In univariate analysis, younger age $(\beta=0.007, p=0.004)$, higher BMI $(\beta=-0.011, p=0.011)$, higher baseline weight $(\beta=-0.004, p=0.004)$, and higher EBWL $(\beta=-0.036$, $p<0.001)$ were significantly associated with greater decrease of TSH after bariatric surgery. Higher baseline TSH $(\beta=-0.439, p<0.001)$ and higher FT3 levels $(\beta=-0.325$, $p<0.001)$ were also associated with greater decrease of TSH after bariatric surgery. In comparison with RYGB, LAGB was associated with a smaller TSH decrease $(\beta=0.286, p<0.001)$, while no significant effect was observed comparing SG and RYGB. On the other hand, sex, the presence of diabetes, dyslipidemia or hypertension, or the systolic and diastolic pressure was not associated with the TSH variation after bariatric surgery (Table 2A).

In a multivariate analysis, after adjusting for relevant covariates, EBWL, baseline BMI, and baseline FT3 remained significantly associated with TSH decrease 12 months after bariatric surgery (Table 2B).

\section{Discussion}

In a population of patients with morbid obesity and normal baseline thyroid function, we observed a significant 
Table 1 Baseline clinical characteristics of the total study population and by TSH groups

\begin{tabular}{|c|c|c|c|c|}
\hline & Total population $(n=949)$ & Normal TSH $(n=718)$ & High-normal TSH $(n=231)$ & $p$ value \\
\hline Sex, $\%$ & & & & 0.001 \\
\hline Male & $13.9 \%$ & $16.0 \%$ & $7.4 \%$ & \\
\hline Female & $86.1 \%$ & $84.0 \%$ & $92.6 \%$ & \\
\hline Age, years & $42.0 \pm 10.3$ & $41.9 \pm 9.7$ & $42.1 \pm 12.0$ & 0.852 \\
\hline Type of surgery & & & & 0.746 \\
\hline Adjustable gastric band & $20.9 \%$ & $21.0 \%$ & $20.3 \%$ & \\
\hline Roux-en-Y gastric bypass & $58.6 \%$ & $58.0 \%$ & $60.6 \%$ & \\
\hline Sleeve gastrectomy & $20.6 \%$ & $21.0 \%$ & $19.1 \%$ & \\
\hline Diabetes, $\%$ & $30.1 \%$ & $30.0 \%$ & $31.6 \%$ & 0.577 \\
\hline Dyslipidemia, \% & $44.1 \%$ & $43.9 \%$ & $44.8 \%$ & 0.818 \\
\hline Hypertension, $\%$ & $60.6 \%$ & $59.4 \%$ & $64.4 \%$ & 0.183 \\
\hline Weight, kg & $116.4 \pm 18.6$ & $116.3 \pm 18.9$ & $116.5 \pm 17.5$ & 0.867 \\
\hline BMI, $\mathrm{kg} / \mathrm{m}^{2}$ & $44.3 \pm 5.7$ & $44.1 \pm 5.5$ & $45.1 \pm 6.0$ & 0.016 \\
\hline Systolic BP, mmHg & $133.3 \pm 17.4$ & $132.4 \pm 17.3$ & $135.9 \pm 17.8$ & 0.011 \\
\hline Diastolic BP, mmHg & $83.0 \pm 11.3$ & $82.9 \pm 11.0$ & $83.4 \pm 12.3$ & 0.575 \\
\hline Waist circumference, $\mathrm{cm}$ & $123.7 \pm 13.3$ & $123.2 \pm 13.2$ & $125.3 \pm 13.3$ & 0.059 \\
\hline Waist-to-hip ratio & $0.93 \pm 0.09$ & $0.93 \pm 0.09$ & $0.93 \pm 0.09$ & 0.661 \\
\hline TSH, mIU/L & $1.97 \pm 0.88$ & $1.57 \pm 0.49$ & $3.23 \pm 0.59$ & $<0.001$ \\
\hline $\mathrm{FT} 4, \mathrm{ng} / \mathrm{dL}$ & $1.05 \pm 0.13$ & $1.05 \pm 0.14$ & $1.04 \pm 0.13$ & 0.115 \\
\hline FT3, pg/mL & $3.20 \pm 0.45$ & $3.15 \pm 0.44$ & $3.35 \pm 0.47$ & $<0.001$ \\
\hline
\end{tabular}

Normal TSH group: TSH level superior or equal to $0.35 \mathrm{mU} / \mathrm{L}$ and below $2.5 \mathrm{mU} / \mathrm{L}$; high-normal TSH group: TSH level superior or equal to $2.5 \mathrm{mU} / \mathrm{L}$ and below or equal to $4.94 \mathrm{mU} / \mathrm{L}$

$B M I$ body mass index, BP blood pressure, TSH thyroid-stimulating hormone, FT4 free thyroxine, FT3 free triiodothyronine

decrease in TSH levels after bariatric surgery. This TSH decrease was associated with postoperative weight loss and was significantly greater in the subgroup of patients with TSH $>2.5 \mathrm{mU} / \mathrm{L}$.
Most [12-16], but not all [17-19], studies evaluating the variation of TSH after bariatric surgery have also shown a decrease of TSH after the procedure. Moulin de Moraes et al. [13] reported that in 54 euthyroid patients submitted to
Fig. 1 TSH variation 12 months after bariatric surgery. Normal TSH group: TSH level superior or equal to $0.35 \mathrm{mU} / \mathrm{L}$ and below $2.5 \mathrm{mU} / \mathrm{L}$; high-normal TSH group: TSH level superior or equal to $2.5 \mathrm{mU} / \mathrm{L}$ and below or equal to $4.94 \mathrm{mU} / \mathrm{L}$. TSH thyroidstimulating hormone

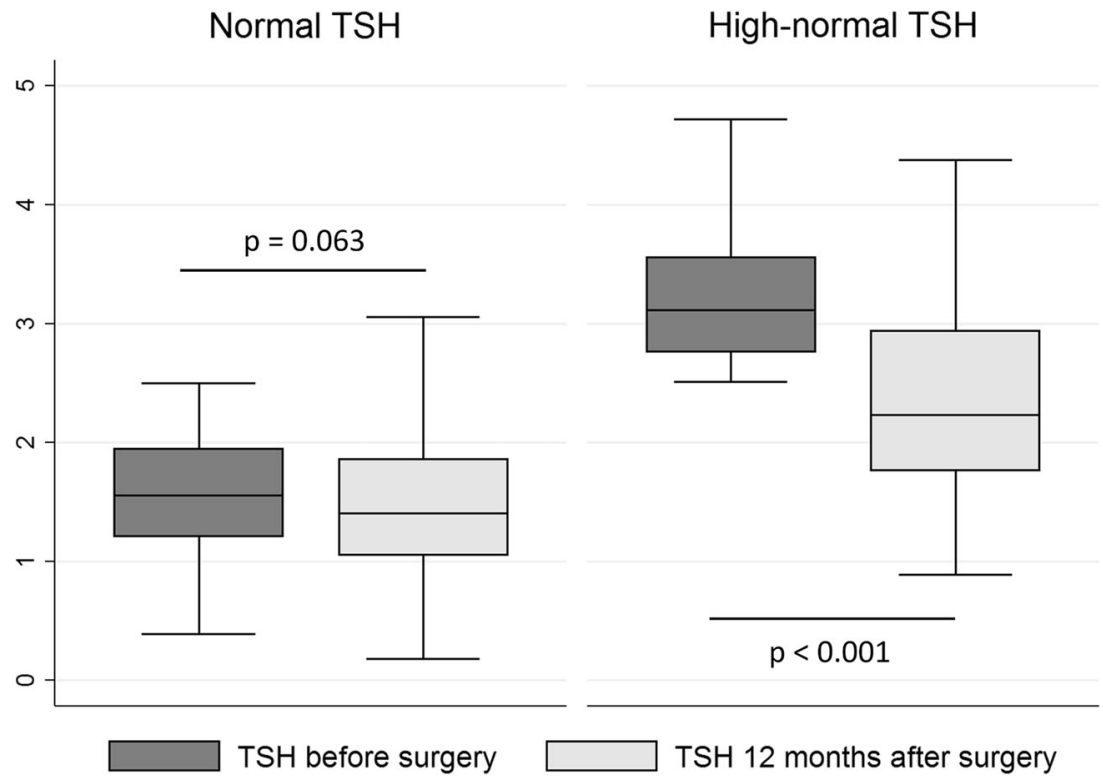


Table 2 TSH variation at 12 months after bariatric surgery

TSH variation 12 months after bariatric surgery

A. Univariate analysis

Excessive body weight loss (10\%)

Sex (female)

Age, years

Type of surgery

Roux-en-Y gastric bypass

Sleeve gastrectomy

Adjustable gastric band

Diabetes

Dyslipidemia

Hypertension

Preoperative weight, $\mathrm{kg}$

Preoperative BMI, $\mathrm{kg} / \mathrm{m}^{2}$

Systolic blood pressure, $\mathrm{mmHg}$

Diastolic blood pressure, $\mathrm{mmHg}$

Waist-to-hip ratio

Preoperative TSH, mIU/L

Preoperative FT4, ng/dL

Preoperative FT3, pg/mL

B. Multivariate analysis

Excessive body weight loss (10\%)

Sex (female)

Age, years

Preoperative BMI, kg/m2

Type of surgery

Roux-en-Y gastric bypass

Sleeve gastrectomy

Adjustable gastric band

Preoperative FT3, pg/mL $\beta$ coefficient

$-0.036(-0.055$ to -0.017$)$

$p$ value

0.09 ( -0.047 to 0.233$)$

$<0.001$

0.007 (0.002 to 0.012$)$

0.193

0.004

(reference category)

-0.068 ( -0.191 to 0.055$)$

0.278

0.286 ( 0.164 to 0.408$)$

$<0.001$

0.014 ( -0.092 to 0.120$)$

0.795

-0.080 ( -0.178 to 0.018$)$

0.111

0.015 ( -0.086 to 0.116$)$

0.769

-0.004 ( -0.006 to -0.001$)$

0.004

-0.011 ( -0.020 to -0.003$)$

0.011

-0.001 ( -0.003 to 0.003$)$

0.831

0.003 ( -0.001 to 0.008 )

0.178

-0.270 ( -0.914 to 0.374$)$

0.410

-0.439 ( -0.487 to -0.391$)$

$<0.001$

0.035 ( -0.328 to 0.399$)$

0.848

-0.325 ( -0.464 to -0.187$)$

$<0.001$

$\beta$ coefficient

$-0.026(-0.051$ to -0.002$)$

$p$ value

0.127 ( -0.050 to 0.304$)$

0.036

0.160

0.003 ( -0.003 to 0.009 )

0.328

$-0.012(-0.023$ to -0.002$)$

0.024

(reference category)

-0.066 ( -0.244 to 0.113$)$

0.471

0.049 ( -0.137 to 0.235$)$

0.605

$-0.246(-0.386$ to -0.107$)$

A: Univariate analysis of TSH variation at 12 months after bariatric surgery. B: Multivariate analysis of TSH variation at 12 months after bariatric surgery BMI body mass index, TSH thyroid-stimulating hormone, FT4 free thyroxine, FT3 free triiodothyronine

RYGB there was a significant decrease in TSH that was not correlated to percent change in BMI and a significant decrease in FT3 but not in FT4. In a study of 38 euthyroid patients submitted to SG, there was also significant decrease in TSH 6-12 months after surgery that was directly related to baseline TSH and not related to EBWL, without significant variation of FT4 [12]. In agreement with our results, a study of 86 patients, not taking medication that could affect the thyroid function, submitted to RYGB or LAGB found a significant decrease of TSH that correlated with the BMI variation [14]. On the other hand, MacCuish et al. (55 patients submitted to RYGB) [17], Alagna et al. (38 patients submitted to biliopancreatic diversion) [18], and Dall'Asta et al. (258 patients submitted to
LAGB) [19] reported an absence of TSH variation after bariatric surgery. The differences between studies may be related to the type of surgery evaluated, the baseline characteristics of the patients or to the statistical power of the studies to detect differences in TSH and to detect associations with EBWL.

Although it was not evaluated in the current study, the variations of FT3 and FT4 levels after bariatric surgery are also relevant to understand the impact of this intervention on the hypothalamus-pituitary-thyroid axis. Most studies [13, 18, $19,22]$ evaluating the effect of bariatric surgery on thyroid hormones showed a significant decrease of FT3. On the other hand, the impact of bariatric surgery on FT4 levels is more controversial with different studies reporting decreasing [22], 
stable $[12-14,18]$, or increasing $[17,19]$ levels after bariatric surgery.

We must highlight that this is the largest study to date evaluating the effect of bariatric surgery on TSH variation in euthyroid patients with morbid obesity and that our evaluation was not restricted to a type of surgery, including three of the most common surgeries for treatment of morbid obesity. According to our results, the decrease of TSH after bariatric surgery is related to the EBWL, and this effect is independent of the type of surgery. As expected, patients submitted to RYGB and SG presented significantly higher EBWL than LAGB. Accordingly, the decrease in TSH levels was significantly smaller in the LAGB group comparing to RYGB and SG (Table 2A). After adjusting to EBWL, the type of surgery was no longer significantly associated with TSH variation (Table 2B), highlighting a more relevant role of weight loss than the type of surgery per se.

In our study, one fourth of the patients had high-normal TSH levels $(>2.5 \mathrm{mU} / \mathrm{L})$. This is in accordance with previous reports of an increase of TSH with increasing body weight [23-25]. Also, in agreement with this, the subgroup with high-normal TSH levels had a higher baseline BMI. The mechanism of TSH elevation in obesity is incompletely understood. The observation that weight loss is associated with decreases in TSH and FT3 has highlighted that TSH elevation may be a consequence and not the cause of obesity $[11-16,18$, 19]. Even in patients with a clinical diagnosis of hypothyroidism, there are reports of decreased levels of TSH after bariatric surgery $[13,14,26,27]$. In two studies, all the patients with untreated subclinical hypothyroidism reached normal values of TSH 12 months after bariatric surgery [13, 14].

The increase of TSH may represent a compensatory activation of hypothalamus-pituitary-thyroid axis in response to excessive body weight [28]. This activation appears to be mediated at least in part by hypothalamic or pituitary effects of leptin [29, 30]. In this study, the observation that FT3 is higher in high-normal TSH group further supports an activation of thyroid axis in morbid obesity.

Interestingly, the patients with higher TSH, those with higher baseline BMI and those with higher FT3 were those that presented a greater decrease of TSH 12 months after surgery. The mechanisms of TSH decrease after bariatric surgery remain incompletely understood and remain to be further elucidated. One of the most plausible explanations is the decrease of leptin levels following bariatric surgery [31]. With decreasing amount of body fat, the decreasing leptin circulating levels [32] reduce the central stimulation of the thyroid axis [29, 30] and promote a decrease of TSH. The observation that this decrease is only significant in the subgroup with baseline $\mathrm{TSH}>2.5 \mathrm{mU} / \mathrm{L}$ suggests that this decrease depends on the baseline overactivation of the thyroid axis. Morbid obesity is known to be a disease with a great heterogeneity of pathophysiological mechanisms and of endocrine adaptations [33] and, probably, only a subgroup of patients present thyroid axis overactivation and consequently TSH decrease after obesity treatment. In summary, bariatric surgery appears to promote a normalization of the thyroid axis function that in the patients with greater baseline dysregulation of the axis will lead to a greater decrease of TSH.

From a clinical point of view, our study highlights that patients with morbid obesity and that present higher TSH levels tend to normalize after bariatric surgery. This points out that caution must be employed when interpreting TSH levels in patients with morbid obesity. Values approaching the upper-limit of the reference interval may not represent a subnormal thyroid function, but a compensatory response of the thyroid axis to morbid obesity. Furthermore, our study emphasizes that the use of narrower reference ranges of serum TSH with a decrease of the upper limit of serum TSH levels, as proposed by several authors [20,21], is not appropriate to the diagnosis of thyroid diseases in patients with morbid obesity.

We must acknowledge some limitations of our work. First, we only evaluated FT3 and FT4 before surgery, and consequently we cannot evaluate the impact of the variation of these parameters on TSH after bariatric surgery. The absence of FT3 and FT4 levels after weight loss hampers interpretation of our findings. Second, we evaluated the variation of TSH using only two moments (before and 12 months after the surgery). As TSH levels may vary with acute illness or other interfering factors, the observed variation in individual patients may also reflect factors other than the effect of weight loss after bariatric surgery. However, as we used a large group of patients, we believe that this fact does not interfere with the interpretation of our results. And third, we only evaluated the effect on TSH levels 12 months after bariatric surgery and so, our results must not be extrapolated to the long-term effects of bariatric surgery on thyroid function. Given that most previous studies have only evaluated the effect on the first 6-24 months on a small number of patients, we believe that future studies with longer follow-up on a large number of patients are important in this field.

\section{Conclusion}

In patients with morbid obesity and normal thyroid function, bariatric surgery promotes a significant decrease of TSH levels 12 months after the procedure. This decrease is significantly greater in patients with baseline high-normal TSH levels and is independently associated with EBWL after bariatric surgery, baseline BMI and baseline FT3 levels. 
Acknowledgments We would like also to acknowledge all the endocrinologists, surgeons, and nutritionists of the Multidisciplinary Group for Surgical Management of Obesity.

\section{Compliance with Ethical Standards}

Conflicts of Interest The authors declare that they have no conflict of interest.

Ethical Approval All procedures performed in studies involving human participants were in accordance with the ethical standards of the institutional and/or national research committee and with the 1964 Helsinki Declaration and its later amendments or comparable ethical standards.

Informed Consent For this type of study formal consent is not required.

Source of Funding No funding was sought for this study.

\section{References}

1. Ng M, Fleming T, Robinson M, et al. Global, regional, and national prevalence of overweight and obesity in children and adults during 1980-2013: a systematic analysis for the Global Burden of Disease Study 2013. Lancet. 2014;384(9945):766-81.

2. Biondi B. Thyroid and obesity: an intriguing relationship. J Clin Endocrinol Metabol. 2010;95(8):3614-7.

3. Buchwald H, Estok R, Fahrbach K, et al. Weight and type 2 diabetes after bariatric surgery: systematic review and meta-analysis. Am J Med. 2009;122(3):248-56. e5

4. Buchwald H, Avidor Y, Braunwald E, et al. Bariatric surgery: a systematic review and meta-analysis. JAMA. 2004;292(14):172437.

5. Chang SH, Stoll CRT, Song J, et al. The effectiveness and risks of bariatric surgery an updated systematic review and meta-analysis, 2003-2012. JAMA Surg. 2014;149(3):275-87.

6. Souteiro P, Belo S, Neves JS, et al. Preoperative beta cell function is predictive of diabetes remission after bariatric surgery. Obes Surg 2016.

7. Mullur R, Liu YY, Brent GA. Thyroid hormone regulation of metabolism. Physiol Rev. 2014;94(2):355-82.

8. Knudsen N, Laurberg P, Rasmussen LB, et al. Small differences in thyroid function may be important for body mass index and the occurrence of obesity in the population. J Clin Endocrinol Metab. 2005;90(7):4019-24.

9. Bjergved L, Jørgensen T, Perrild $\mathrm{H}$, et al. Thyroid function and body weight: a community-based longitudinal study. PLoS One. 2014;9(4):e93515.

10. Rotondi M, Leporati P, La Manna A, et al. Raised serum TSH levels in patients with morbid obesity: is it enough to diagnose subclinical hypothyroidism? Eur J Endocrinol. 2009;160(3):403-8.

11. Michalaki MA, Vagenakis AG, Leonardou AS, et al. Thyroid function in humans with morbid obesity. Thyroid. 2006;16(1):73-8.

12. Abu-Ghanem $Y$, Inbar R, Tyomkin $V$, et al. Effect of sleeve gastrectomy on thyroid hormone levels. Obes Surg. 2015;25(3):452-6.

13. Moulin de Moraes CM, Mancini MC, de Melo ME, et al. Prevalence of subclinical hypothyroidism in a morbidly obese population and improvement after weight loss induced by Rouxen-Y gastric bypass. Obes Surg. 2005;15(9):1287-91.

14. Chikunguwo S, Brethauer S, Nirujogi V, et al. Influence of obesity and surgical weight loss on thyroid hormone levels. Surg Obes Relat Dis. 2007;3(6):631-5.

15. Camastra S, Manco M, Frascerra S, et al. Daylong pituitary hormones in morbid obesity: effects of bariatric surgery. Int J Obes. 2009;33(1):166-72.

16. Janković D, Wolf $\mathrm{P}$, Anderwald $\mathrm{C}-\mathrm{H}$, et al. Prevalence of endocrine disorders in morbidly obese patients and the effects of bariatric surgery on endocrine and metabolic parameters. Obes Surg. 2012;22(1):62-9.

17. MacCuish A, Razvi S, Syed AA. Effect of weight loss after gastric bypass surgery on thyroid function in euthyroid people with morbid obesity. Clin Obes. 2012;2(1-2):25-8.

18. Alagna S, Cossu ML, Masala A, et al. Evaluation of serum leptin levels and thyroid function in morbidly obese patients treated with bariatric surgery. Eat Weight Disord. 2003;8(2):95-9.

19. Dall'Asta C, Paganelli M, Morabito A, et al. Weight loss through gastric banding: effects on TSH and thyroid hormones in obese subjects with normal thyroid function. Obesity. 2010;18(4):854-7.

20. Wartofsky L, Dickey RA. The evidence for a narrower thyrotropin reference range is compelling. J Clin Endocrinol Metab. 2005;90(9):5483-8.

21. Baloch Z, Carayon P, Conte-Devolx B, et al. Laboratory medicine practice guidelines. Laboratory support for the diagnosis and monitoring of thyroid disease. Thyroid. 2003;13(1):3-126.

22. Di J, Zhang H, Yu H, et al. Effect of Roux-en-Y gastric bypass on the remission of type 2 diabetes: a 3 -year study in Chinese patients with a BMI $<30 \mathrm{~kg} / \mathrm{m} 2$. Surg Obes Relat Dis. 2016;12(7):1357-63.

23. de Moura Souza A, Sichieri R. Association between serum TSH concentration within the normal range and adiposity: a review. Eur J Endocrinol. 2011;165(1):11-5.

24. Nyrnes A, Jorde R, Sundsfjord J. Serum TSH is positively associated with BMI. Int J Obes. 2006;30(1):100-5.

25. Fox CS, Pencina MJ, D'Agostino RB, et al. Relations of thyroid function to body weight: cross-sectional and longitudinal observations in a community-based sample. Arch Intern Med. 2008;168(6): 587-92.

26. Fazylov R, Soto E, Cohen S, et al. Laparoscopic Roux-en-Y gastric bypass surgery on morbidly obese patients with hypothyroidism. Obes Surg. 2008;18(6):644-7.

27. Janssen IM, Homan J, Schijns W, et al. Subclinical hypothyroidism and its relation to obesity in patients before and after Roux-en-Y gastric bypass. Surg Obes Relat Dis. 2015;11(6):1257-63.

28. Reinehr T. Obesity and thyroid function. Mol Cell Endocrinol. 2010;316(2):165-71.

29. Kok P, Roelfsema F, Frölich M, et al. Spontaneous diurnal thyrotropin secretion is enhanced in proportion to circulating leptin in obese premenopausal women. J Clin Endocrinol Metabol. 2005;90(11):6185-91.

30. Betry C, Challan-Belval MA, Bernard A, et al. Increased TSH in obesity: evidence for a BMI-independent association with leptin. Diabetes Metab. 2015;41(3):248-51.

31. Kruljac I, Mirošević G, Kirigin LS, et al. Changes in metabolic hormones after bariatric surgery and their predictive impact on weight loss. Clin Endocrinol. 2016;85(6):852-60.

32. Park HK, Ahima RS. Physiology of leptin: energy homeostasis, neuroendocrine function and metabolism. Metabolism. 2015;64(1):24-34.

33. Tchernof A, Després JP. Pathophysiology of human visceral obesity: an update. Physiol Rev. 2013;93(1):359-404. 